報文 3

\title{
Size Measurement of Microparticles in Liquids Using Photoacoustic Spectroscopy
}

\author{
Takehiko Kitamori, Kazumichi SuzUKI, Tsuguo SAwadA* \\ and Yohichi GoHsH*
}

Energy Research Laboratory, Hitachi, Ltd., 1168

Moriyama, Hitachi, Ibaraki 316, JAPAN

* Department of Industrial Chemistry, Faculty of Engineering, University of Tokyo, 7-3-1 Hongo, Bunkyo, Tokyo 113, JAPAN

(Received July 7, 1986)

\author{
北森 武彦・藤井 正昭・沢田 嗣郎 ·合志 陽一 \\ 陎日立製作所エネルギー研究所 日立市森山町 1168（テ316） \\ 東京大学工学部工業化学科文京区本郷 7-3-1（干113）
}

(1986年 7 月 7 日受理)

\begin{abstract}
The size measurement method for microparticles in liquids using the phase shift of photoacoustic signals theorized in our earlier paper [J. Appl. Phys., 58, 1456 (1985)] was verified using polystyrene turbid solutions. The experimental values for polystyrene microparticles, radii $0.4-5.2 \mu \mathrm{m}$, agreed with the theoretical values within the experimental accuracy of $\pm 0.2 \mathrm{deg}$. The physical meaning of the phase shift was clarified as representing the delay angle of the photoacoustic signal generation determined by the ratio of the heat release time from the microparticles to the modulation period of the excitation beam. Applications of photoacoustic spectroscopy for sizing and determination of microparticles in liquids by proper use of modulation frequency are discussed.
\end{abstract}

\section{Introduction}

Photoacoustic spectroscopy (PAS) has been applied to liquid phase samples as a direct coupling method, in which a piezoelectric signal detector is directly contacted with the liquid sample ${ }^{1,2)}$. Using a laser as the beam source, the method is quite sensitive, and it has been applied as a weak absorption detector ${ }^{3)}$. The lower limit for a detectable absorption coefficient is on the order of $10^{-8} \mathrm{~cm}^{-1}$, utilizing an optimized cylindrical cell ${ }^{4}$. Thus PAS has been effectively applied to ultrasensitive colorimetric analysis in ppt $(\mathrm{pg} / \mathrm{mL})$ regions $^{5-9)}$. These applications are based on measurement of the photoacoustic (PA) signal amplitude. However, the phase of the PA signal has not been applied to characterization of liquid samples.

In our previous papers, the phase of the PA signal in liquids was analyzed using linear response theory, and the relationship between the phase and energy migration in liquid was clarified $^{10,11)}$. This generalized theory was applied to the phase due to heat transfer from microparticle to the liquid medium, and the possibilities of sizing and thermal characterizations for suspended microparticles by phase measurements were indicated. Part of this characterization method, i.e. measurement of 
heat transfer coefficients, was proved $^{11)}$. In this paper, the sizing method is verified for sub-micron to micron particles, and the relationships between the PA signals, heat migration time from microparticles to the medium, and modulation frequency of the excitation beam are discussed. Analytical applications of PAS for sizing and determination of microparticles in liquids are also introduced.

\section{Experimental}

\subsection{Outline of the method}

The basic relationships between the phase of PA signals and the size of microparticles in liquids was obtained from analysis of rigorous thermal and acoustic coupling equations for the following process $:^{10,11)}$ the temperature of the particles rises with the heat caused by absorption of the excitation beam; heat in the particles is released to the medium as a heat flux along the temperature field; the heat distribution in the medium fluctuates due to intensity modulation of the excitation beam; an acoustic wave is generated by the fluctuation of the heat distribution in the medium. The thermal process, in which the photoenergy of the excitation beam is converted to the heat distribution in the medium, is described by thermal equations for the particles and the medium. The acoustic process in which the PA signal is generated by fluctuation of the heat distribution is described by the wave equation for which the source term is the fluctuation of the heat distribution. The phase of the PA signal can be obtained from the frequency domain solutions of these coupling equations using linear response theory, and the phase $\phi_{D}$ due to the thermal process is described as follows ; ${ }^{10,11)}$

$$
\begin{aligned}
\phi_{D} & =\tan ^{-1} \frac{\sigma_{s} C_{p_{s}} V}{S h} \omega_{0} \\
& =\tan ^{-1} \frac{\sigma_{s} C_{p_{s}} d^{2}}{3 \kappa} \omega_{0}
\end{aligned}
$$

(for spherical particles)

where $\sigma_{s}, C_{p_{s}}$ and $d$ are the density, specific heat, and the radius of the particles, respective$1 \mathrm{y} ; \kappa$ is the heat conductance of the medium; $h$ is the heat transfer coefficient from the particles to the medium; and $\omega_{0}$ is the angular frequency of the beam modulation. $S$ and $V$ represent the surface area and the volume of the particle, respectively. The physical meaning of $\phi_{D}$ is discussed later.

In addition to the phase $\phi_{D}$, the measured phase $\phi_{m}$ has other phase components $\phi_{Q}$ and $\phi_{R}$ due to propagation of the PA signal and response of the PAS equipment, respectively. The phase $\phi_{Q}$ can be obtained from the analysis of the acoustic process, and it corresponds to the phase of the PA signals generated in a solvent itself ${ }^{12)}$. Therefore, in the experiment, the value of the additional phase $\phi_{Q}+\phi_{R}$ can be obtained as the background phase $\phi_{B}$ by measuring the PA signal phase of the solvent. Hence, the measured phase $\phi_{m}$ for a turbid solution and background phase $\phi_{B}$ are written as follows,

$$
\begin{aligned}
& \phi_{m}=\phi_{D}+\phi_{Q}+\phi_{R}, \\
& \phi_{B}=\phi_{Q}+\phi_{R} .
\end{aligned}
$$

Then, $\phi_{D}$ can be obtained as $\phi_{m}-\phi_{B}$.

\subsection{Measurements}

The dependence of the phase $\phi_{D}$ on the radius of the microparticles was measured with polystyrene turbid solutions of different particle radii. The radii were $0.4,0.5,1.2$, 3.2 and $5.2 \mu \mathrm{m}$. Turbid solutions with $1 \mathrm{ppm}$ solid were prepared by stepwise dilutions of standard stock solutions of $10 \%$ solid using distilled and demineralized water filtered

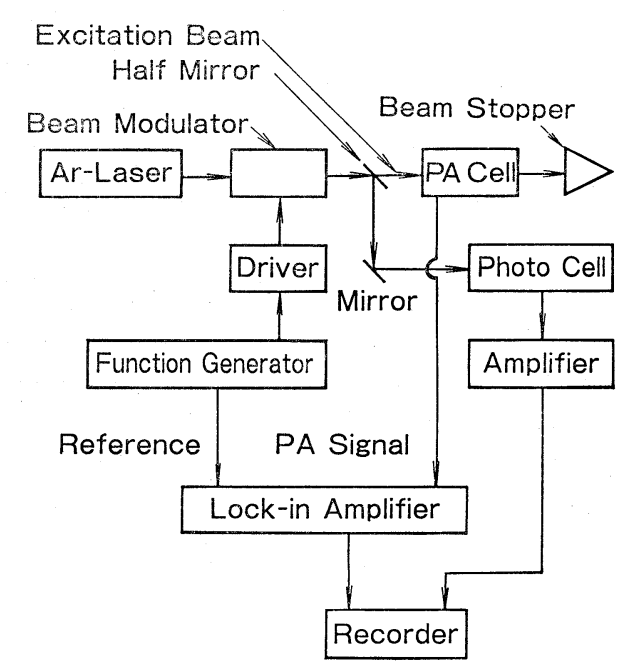

Fig. 1 A block diagram of the PAS apparatus. 
Table 1 Experimental parameters for measurement of the phase shifts.

\begin{tabular}{cc}
\hline \multicolumn{1}{c}{ Parameter } & Value \\
\hline Cell & \\
Cell radius & $4.5 \mathrm{~mm}$ \\
Cell length & $100 \mathrm{~mm}$ \\
Excitation beam & \\
Wavelength & $514.5 \mathrm{~nm}$ \\
Beam power & $200 \mathrm{~mW}$ \\
Beam radius & $2.0 \mathrm{~mm}$ \\
Modulation frequency & $6.5 \mathrm{kHz}$ \\
Modulation function & Sinusoidal \\
\hline
\end{tabular}

through $0.1 \mu \mathrm{m}$ membrane filters. A portion of each turbid solution was filtered through the $0.1 \mu \mathrm{m}$ membrane filter, and the particle radii were measured by observation with a scanning electron microscope.

A block diagram of the PAS equipment is shown in Fig. 1, and the experimental parameters are listed in Table 1 . A cylindrical direct coupling cell for which the signal detector was a cylindrical piezoelectric transducer (PZT) was used ${ }^{4}$. The excitation beam was sinusoidally modulated at $6.5 \mathrm{kHz}$ by the acousto-optic modulator. The beam power was $200 \mathrm{~mW}$, which was low enough to avoid generation of PA signals on PZT surface due to the direct exposure to the scattered beam.

The background phase $\phi_{B}$ was measured with the water subjected to the following ultrapurification steps: distillation in a glass apparatus, two demineralizations with a resin ion exchanger, and filteration through an activated carbon filter and a membrane filter. The resistivity of the obtained water was about $18 \mathrm{M} \Omega / \mathrm{cm}$, and particulate impurities above $0.1 \mu \mathrm{m}$ were almost completely removed, hence the PA signal generated from any impurities was negligibly small in comparison with that of the water. The phase $\psi_{m}$ for each polystyrene turbid solution and the background phase $\phi_{B}$ for the water were measured. The phase $\phi_{D}$ for each radius was calculated as $\phi_{m}-\psi_{B}$. The results obtained are plotted in Fig. 2.

\section{Discussion}

The theoretical values of the phase shift for the polystyrene microparticle are calculated

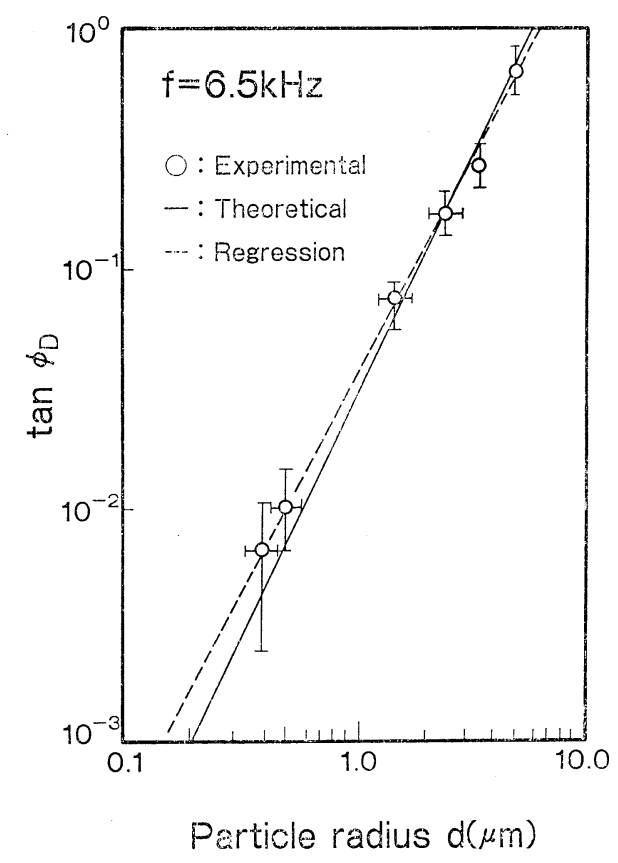

Fig. 2 Dependence of the phase shift on the radius of polystyrene microparticles in liquids. The solid line is the theoretical values calculated with eq. (2), and the dashed line is the regression line for the measured values. The experimental parameters are listed in Table 1, and the material constants for the calculation are listed in Table 2.

Table 2 Material constants.

\begin{tabular}{|c|c|}
\hline Parameter & Value \\
\hline \multicolumn{2}{|l|}{ Polystyrene } \\
\hline Specific heat; $C_{p_{S}}$ & $1.22 \times 10^{3} \mathrm{~J} / \mathrm{kgK}$ \\
\hline Density $; \sigma_{s}$ & $1.05 \times 10^{3} \mathrm{~kg} / \mathrm{m}^{3}$ \\
\hline \multicolumn{2}{|l|}{ Water } \\
\hline Heat conductance; $\kappa$ & $6.3 \times 10^{-1} \mathrm{~J} / \mathrm{msK}$ \\
\hline \multicolumn{2}{|l|}{$\mathrm{BaSO}_{4}$} \\
\hline Specific heat; $C_{p_{s}}$ & $4.36 \times 10^{2} \mathrm{~J} / \mathrm{kgK}$ \\
\hline Density; $\sigma_{s}$ & $4.49 \times 10^{3} \mathrm{~kg} / \mathrm{m}^{3}$ \\
\hline
\end{tabular}

with eq. (2) using the parameters listed in Table 2, and the values are indicated in Fig. 2 by the solid line. The experimental results agree well with the theoretical values within the experimental accuracy and the size distributions of each solution. Hence the relationship between the phase shift of PA signals and the radius of the microparticles in liquids, 
expressed in eq. (2), is confirmed, and the size measurement based on the relationship is verified. However, the slope of the regression line in the graph is smaller than that of the theoretical values. This seems to be due to approximating the heat transfer coefficient $h=$ $\kappa / d$ in eq. (1), in which additional terms with respect to the particle radius $d$ originating in the Grashof number are neglected ${ }^{10,13)}$.

The phase measurement accuracy of the present PAS equipment is $\pm 0.2 \mathrm{deg}$, hence the lower limit of the size measurement defined as twice the experimental accuracy is calculated as $d=0.48 \mu \mathrm{m}$ using eq. (2). In Fig. 2, the experimental values at $d=0.4$ and $0.5 \mu \mathrm{m}$, including their error bars are overlapped, and closely approach the lower sizing limit. Equation (2) shows that the tangent of the phase shift $\phi_{D}$ is proportional to the modulation frequency $\omega_{0}$, as was also verified previously $^{10)}$. According to this relationship, the phase shift can be enlarged by using a higher modulation frequency, and the size measurement limit can be lowered. However, a more sensitive PAS system is required for using higher modulation frequency than $6.5 \mathrm{kHz}$ at which the present experiment was carried out. Because the PA signal magnitude decreases with increased modulation frequency, ${ }^{12)}$ and the signal magnitude became insufficient for the phase measurement over $6.5 \mathrm{kHz}$ modulation frequency region with the present PAS system.

To clarify the physical meanings of the phase shift, eq. (1) is rewritten as

$$
\phi_{D}=\tan ^{-1} \frac{\tau}{\tau_{f}} 2 \pi
$$

where

$$
\begin{aligned}
\tau & =\frac{\sigma_{s} C_{p_{s}} V}{S h}, \\
\tau_{f} & =\frac{1}{f},
\end{aligned}
$$

and $f\left(\omega_{0}=2 \pi f\right)$ is modulation frequency. The denominator of eq. (4) means the heat release quantity per unit time and unit temperature difference between the particles and the medium, while the numerator is the heat capacity of the particles. Hence $\tau$ means the time re- quired for heat release from the particles to the medium. The phase shift means the delay angle of the PA signal generation determined by the ratio between the heat release time $\tau$ and the modulation period $\tau_{f}$. The PA signals $P(\vec{r}, t)$ from the microparticles in liquids are written as follows ${ }^{11)}$ using $\tau$ and $\tau_{f}$,

$$
\begin{aligned}
& P(\vec{r}, t)=\eta I_{0} \widetilde{D}(\tau) Q(\vec{r}, t) \\
& \eta=\frac{\beta}{C_{p}} \frac{\kappa}{\kappa_{s}} \alpha_{s} V N_{s} \\
& =\frac{\beta}{C_{p}} \frac{\kappa}{\kappa_{s}} \alpha_{s} \frac{C_{s}}{\sigma_{s}}, \\
& Q(\vec{r}, t)=\mathrm{e}^{i \omega_{0} t} i \omega_{0} \int_{\overrightarrow{r^{\prime}}} R\left(\vec{r}^{\prime}\right) G_{\omega_{0}}\left(\vec{r} \mid \vec{r}^{\prime}\right) d \vec{r}^{\prime}, \\
& \widetilde{D}(\tau)=\frac{1}{\frac{\tau}{\tau_{f}} i+1},
\end{aligned}
$$

where $C_{p}$ is the specific heat; $\beta$ is the isothermal expansion coefficient ; $\alpha$ is the absorption coefficient; $N$ is the number density; and $C$ is the concentration. The subscript $s$ identifies the material constants for microparticles. $I_{0}$ is the excitation beam power and the function $R(\vec{r})$ is its distribution, and $G_{\omega_{0}}\left(\vec{r} \mid \vec{r}^{\prime}\right)$ is the propagator. Hence the function $Q(\vec{r}, t)$ means the PA signal generation and propagation in the medium. On the other hands, $\widetilde{D}(\tau)$ is the Fourier image of the impulse response concerned with formation of the heat distribution in the medium, and it expresses the influence of the heat migration process on the signal generation. The phases $\phi_{D}$ and $\phi_{Q}$ can be obtained as the argument of $\widetilde{D}(\tau)$ and $Q(\vec{r}, t)$ respectively. When the modulation frequency is low enough and $\tau_{f}$ is sufficiently larger than $\tau$, the value of $\widetilde{D}(\tau)$ becomes 1 , and eq. (6) shows that the magnitude of the PA signal does not depend on the particle radius. Therefore, when the modulation period $\tau_{f}$ is sufficiently larger than $\tau$, the PA signal is proportional to the concentration as shown in eq. (7), and the calibration curve does not depend on the particle size. Oda et al. ${ }^{14)}$ give experimental evidence for these theoretical conclusions in their measurements of the calibration curves for $\mathrm{BaSO}_{4}$ (particle radii : 0.3-11 $\mu \mathrm{m}$; modulation frequency: $33 \mathrm{~Hz}$ ). Using eq. (4) and the parameters listed in Table 2, $\tau$ for 
these $\mathrm{BaSO}_{4}$ particles is calculated to be 0.1 $139.2 \mu \mathrm{s}$, and the value of $\tau_{j}$ is $3.0 \times 10^{-2} \mathrm{~s}$. Hence, in their experiments $\tau$ was small enough for the approximation $\widetilde{D}(\tau) \simeq 1$ to be valid.

In summarizing the above discussion, PAS can size microparticles in liquids and determine their concentrations, by properly selecting a modulation frequency with regard to the heat release time of the microparticles. Therefore, PAS is expected to be a useful tool for study of colloidal and turbid solutions.

\section{Conclusion}

A size measurement method for microparticles in liquids using the phase shift of the PA signals, which is caused by the time required for heat release from the microparticles to the medium, was proved. The phase shifts were measured at the modulation frequency of $6.5 \mathrm{kHz}$, with polystyrene turbid solutions having particle radii of $0.4-5.2 \mu \mathrm{m}$. The experimental results agreed well with the theoretical values. The phase measurement accuracy of the present PAS equipment was $\pm 0.2 \mathrm{deg}$, and the lower limit of this sizing method, defined by doubling the accuracy value was $0.48 \mu \mathrm{m}$ of radius for the present conditions. However, the tangent of the phase shift is proportional to the modulation frequency, and the limitation can be improved by using higher modulation frequencies.

The physical meaning of the phase shift was shown to represent the delay angle of PA signal generation determined by the ratio between the heat release time and the modulation period of the excitation beam. When the modulation frequency was low enough, the delay angle of the PA signal generation was negligibly small and the PA signal magnitude was proportional to the concentration of the microparticles independent of their sizes. Therefore, PAS can size microparticles in liquids and determine their concentrations, by properly selecting the modulation frequency with regard to their heat release time.

\section{References}

1) T. Sawada and T. Kitamori: Physical Acoustics Vol. 23 (Academic Press, New York, to be published in 1986)

2) C. K. N. Patel and A.C. Tam: Rev. Mod. Phys. 53, 517 (1983)

3) D. S. Kliger, ed.: Ultrasensities Laser Spectroscopy (Academic Press, New York, 1983)

4) T. Kitamori, M. Fujii, T. Sawada and Y. Gohshi: J. Spectrosc. Soc. Jpn. 34, 359 (1985)

5) W. Lahmann, H. Ludewing and H. Welling: Anal. Chem. 49, 549 (1977)

6) S. Oda, T. Sawada and H. Kamada: Anal. Chem. 50, 865 (1978)

7) T. Sawada and S. Oda: Anal. Chem. 53, 539 (1981)

8) S. Oda and T. Sawada: Anal. Chem. 53, 471 (1981)

9) T. Kitamori, T. Sawada, K. Suzuki, Y. Gohshi and K. Motojima: Anal. Chem., 58, 2275 (1986)

10) T. Kitamori, M. Fujii, T. Sawada and Y. Gohshi: J. Appl. Phys. 58, 1456 (1985)

11) T. Kitamori, M. Sakagami, T. Sawada and Y. Gohshi: Jpn. J. Appl. Phys. 24, Sup. 24-1, 210 (1985)

12) T. Kitamori, M. Fujii, T. Sawada and Y. Gohshi: J. Appl. Phys. 55, 4005 (1984)

13) W.E. Ranz: Chem. Engng. Progr. 48, 141 (1952)

14) S. Oda, T. Sawada, T. Moriguchi and H. Kamada: Anal. Chem. 52, 650 (1980) 